\title{
The ISBER 2019 Awards
}

\author{
Kristina Hill ${ }^{1,2}$ and Zisis Kozlakidis ${ }^{3,4}$
}

$\mathrm{T}$ he International Society for Biological and Environmental Repositories (ISBER) will confer a number of different awards at its annual meeting this year in Shanghai, China. These include the ISBER travel awards, as well as a number of awards celebrating the achievements and contributions of individuals from a wide range of professional backgrounds and geographical areas who have made a significant contribution to ISBER and the biobanking field in general.

\section{The ISBER Travel Award}

The ISBER travel award was established $>10$ years ago and has made it possible for 13 individuals from low to middle income countries to attend the ISBER annual meeting. ${ }^{1}$ The travel award is targeted toward individuals interested in either establishing or already managing a biorepository in their particular country or region. ISBER hopes that awardees will gain useful information from the various symposiums and workshops, network with experienced leaders in the biobanking field, and subsequently bring back valuable information from the meeting to help inform their colleagues and home country. The ISBER Education \& Training committee reviews the applications and awardees are provided with airfare, hotel, a daily stipend, and complimentary attendance to the meeting event gala and $5 \mathrm{~K}$ Fun Walk/Run.

ISBER is able to fund one applicant's attendance to the 2019 ISBER annual meeting in Shanghai, China. There were eight eligible applicants representing the countries of Rwanda, Ghana, the Philippines, Uganda, Gambia, and the Ukraine. Dr. Anna Piddubna, assistant professor at Sumy State University in Sumy, Ukraine, was chosen as the 2019 ISBER travel award recipient. Dr. Piddubna supervises the storage of infectious disease biological samples at the University's Laboratory of Molecular and Genetic Studies biobank. Dr. Piddubna hopes attendance at the ISBER annual meeting will benefit her goal of establishing a Ukrainian national biobank. These benefits will include exposure to the most up-to-date standards and practices by attending the various talks, poster sessions, and workshops in addition to networking with top experts in the biobanking field. After the meeting, Dr. Piddubna hopes to develop and deliver a proposal for the creation of a national biobank, develop and implement innovations for applications at regional institutional biorepositories, organize seminars for biorepository personnel, and deliver a wide range of lectures to university students. ISBER is very excited to aid Dr. Piddubna in these achievements!

\section{The ISBER Annual Awards}

The ISBER award for outstanding achievement in biobanking, sponsored by Worthington Industries, is designed to recognize individuals who have made outstanding contributions to the field of biobanking. The award can be given for a single outstanding achievement or for a life-time body of outstanding work in the field.

ISBER is proud to announce that the 2019 winner of the ISBER outstanding achievement in biobanking award is Marianna Bledsoe. Marianna J. Bledsoe, MA, is an independent consultant and deputy editor for the journal Biopreservation and Biobanking. As a former senior program manager for Biorepositories and Biobanking at the Department of Veterans Affairs (VA), Office of Research and Development, she provided strategic direction and oversight of the VA's tissue banking activities. Before this position, she was deputy associate director of the Clinical Research Policy Analysis and Coordination Program in the Office of Science Policy, Office of the Director, National Institutes of Health. She served as ISBER President (2007-2008) and established the ISBER awards program. She has represented ISBER on numerous conferences and meetings globally, and has served on many ISBER committees. She has served as ISBER liaison to Public Responsibility in Medicine and Research (PRIM\&R) and has organized and taught numerous PRIM\&R tissue banking workshops and programs for the research ethics community. She continues to offer her leadership, strong voice, and insights in the realm of biobanking, especially on ethical, legal, and social issues challenges. ${ }^{2}$ For her work, she received the ISBER distinguished leadership and services award in 2012. In 2011 she was instrumental in recognizing the need for a forum to provide ISBER's membership with a voice in shaping the

${ }^{1}$ Eastern Virginia Medical School, Norfolk, Virginia.

${ }^{2}$ Chair, ISBER Education and Training Committee.

${ }^{3}$ International Agency for Research on Cancer (IARC), World Health Organization (WHO), Lyon, France.

${ }^{4}$ Chair, ISBER Nominating Committee. 
evolving regulations and policies affecting biobanking and biospecimen use across the globe. As a result, the ISBER board of directors established the ISBER science policy advisory committee in 2011 with global ISBER member representation, creating an active communication forum between biobankers, ethicists, legal professionals, and policy makers. Under Marianna's leadership, the ISBER science policy committee has contributed $>15$ policy comments and/or recommendations to national organizations globally.

The ISBER founder's award, sponsored by Chart MVE, recognizes individuals who have provided outstanding leadership to the founding, support, and incorporation of ISBER as an international biobanking society. ISBER is proud to announce Prof. William E. Grizzle as the 2019 recipient of the ISBER founder's award. Prof. Grizzle is professor at the Department of Pathology at the University of Alabama at Birmingham (UAB), AL. He is a board certified anatomic and clinical pathologist. He has been principal investigator of the Southern Division of the Cooperative Human Tissue Network since 1987 and director of the Tissue Resource Facility at the UAB since 1983 . He is internationally recognized as one of the leading authorities in human tissue resources and has published extensively in this area. ${ }^{3} \mathrm{He}$ is a founding member of the ISBER, has served as ISBER president (2005-2006), and has been instrumental in creating and disseminating educational material for biobanking. His contribution to biobanking was recognized by the 2015 ISBER outstanding achievement in biobanking award. Prof. Grizzle is an internationally recognized researcher with expertise in oral neoplasia. He was associate director of the UAB Oral Cancer Research Center (1996-2002). He is the current head of the Pathology Program for Translational Research in Neoplasia, Department of Pathology, UAB, and principal investigator of one of the three Validation Laboratories of the Early Detection Research Network.

The ISBER distinguished leadership and service award is designed to honor ISBER members who have demonstrated exceptional leadership to further the mission and goals of the society and/or significant long-standing contributions to the society. ISBER is proud to announce Dr. Daniel SimeonDubach as the 2019 recipient of the ISBER distinguished leadership and service award. Daniel Simeon-Dubach, MD, MHA, is the chair of the ISBER Standards Committee and the owner of Medservice, a biobanking consulting and service, located in Walchwil, Switzerland. Having established a successful career in the medical and pharmaceutical fields, Dr. Simeon-Dubach has worked in biobanks for $>12$ years. His focus is on biobank business planning, governance and sustainability, biobanking standards, and collaboration between academia and the biomedical industry. He was coorganizer and cochair of several symposia on sustainability at ISBER annual meetings and other meetings in the past.
He has co-organized the first global biobank business planning survey. ${ }^{4} \mathrm{He}$ organizes workshops to create a biobank business plan and coaches biobanks in creating these business plans. He has been a member of the ISBER organizing advisory committee for many years. As the chair of the ISBER standards committee, he has overseen the successful launch of the fourth edition of the ISBER Best Practices for Repositories, 5 the ensuing translations, and updates to ISBER best practices, and develops tools to improve and standardize modern biobanking. He is section editor for biobanking management of Biopreservation and Biobanking and on the editorial board of "Synergy."

The ISBER special service awards recognize individuals who have made exceptional contributions toward the goals of the society through the performance of a special service or act on behalf of the organization. ISBER is proud to announce the following recipients of the 2019 ISBER special services award:

Alison Parry-Jones, for her contribution to the ISBER Regional Ambassadors program.

Catherine Seiler, for her contribution to the ISBER Communications Committee.

Monique Albert, for her efforts to support ISBER in Latin America and the development of Self-Assessment Tools.

William Mathieson, for his efforts to promote Biospecimen Science Research.

Xiaoyan Zhang, for her efforts in supporting closer links between ISBER and BBCMBA.

\section{References}

1. Seiler CY. Where are they now? ISBER Travel Award Winners 2014-2017. Biopreserv Biobank 2017;15:487-489.

2. Bledsoe MJ. Ethical legal and social issues of biobanking: Past, present, and future. Biopreserv Biobank 2017;15:142-147.

3. Grizzle WE, Sexton KC, McGarvey D, Menchhofen ZV, LiVolsi V. Lessons learned during three decades of operations of two prospective bioresources. Biopreserv Biobank 2017;16:483-492.

4. Simeon-Dubach D, Henderson MK. Sustainability in biobanking. Biopreserv Biobank 2014;12:287-291.

5. Simeon-Dubach D, Kozlakidis Z. New standards and updated best practices will give modern biobanking a boost in professionalism. Biopreserv Biobank 2018;16:1-2.

Address correspondence to: Zisis Kozlakidis, PhD, MBA, FLS

International Agency for Research on Cancer (IARC) World Health Organization (WHO) 150 Cours Albert Thomas 69372 Lyon, CEDEX 08 France

E-mail: kozlakidisz@iarc.fr 